\title{
Computation of invariant curves in the analysis of periodically forced neural oscillators
}

\author{
Alberto Pérez-Cervera, Gemma Huguet, and Tere M-Seara
}

\begin{abstract}
Background oscillations, reflecting the excitability of neurons, are ubiquitous in the brain. Some studies have conjectured that when spikes sent by one population reach the other population in the peaks of excitability, then information transmission between two oscillating neuronal groups is more effective. In this context, phase locking relationships between oscillating neuronal populations may have implications in neuronal communication as they assure synchronous activity between brain areas. To study this relationship, we consider a population rate model and perturb it with a time-dependent input. We use the stroboscopic map and apply powerful computational methods to compute the invariant objects and their bifurcations as the perturbation parameters (frequency and amplitude) are varied. The analysis performed shows the relationship between the appearance of synchronous and asynchronous regimes and the invariant objects of the stroboscopic map.
\end{abstract}

Key words: synchronization, phase locking, stroboscopic map, invariant curves, rotation number

\footnotetext{
A. Pérez-Cervera

Dept. Matemàtica Aplicada, Universitat Politècnica de Catalunya, Avda. Diagonal 647 (ETSEIB), E-08028, Barcelona, Spain e-mail: alberto.perez@upc.edu

G. Huguet

Dept. Matemàtica Aplicada, Universitat Politècnica de Catalunya, Avda. Diagonal 647 (ETSEIB), E-08028, Barcelona, Spain e-mail: gemma.huguet@upc.edu

T. M-Seara

Dept. Matemàtica Aplicada, Universitat Politècnica de Catalunya, Avda. Diagonal 647 (ETSEIB), E-08028, Barcelona, Spain e-mail: tere.m-seara@.upc.edu
} 


\section{Introduction}

Since they were first observed in 1929 by Hans Berger [2], neural oscillatory activity has been a topic of great interest and debate in neuroscience which extends until nowadays because its functional role it is not completely understood. Although oscillatory behaviour can occur across different mechanisms, this work will focus on oscillations generated by neuronal ensembles or populations.

Neurons are the fundamental cells within the brain, and they basically communicate through electrical impulses [5]. Neurons integrate inputs from neighbouring neurons across dendrites. When these inputs cause the neuron membrane potential to reach a certain critical value or threshold, the neuron will respond with a characteristic voltage change of large amplitude and short time duration $(\approx 1 \mathrm{~ms})$ known as action potential or spike. This action potential then travels along the axon of the neuron to the axon terminal, where its effect will be felt by the other neurons which this neuron is connected. Neurons can be excitatory or inhibitory, depending on the effect of the connection (synapse) on the receiving neuron (synapses): inputs from an excitatory (inhibitory) neuron depolarize (hyperpolarize) the membrane potential of the receiving neuron.

We consider a neuronal population consisting of excitatory and inhibitory neurons. Firing patterns of excitatory neurons can synchronize across inhibitory action, since inhibition can create windows of opportunity where excitatory neurons can fire [15], thus, showing oscillatory activity.

As the brain is such a highly connected device, the activity of an oscillatory population affects the activity of other populations to which is connected. A simplified framework for this setting is to consider the effect of an oscillatory input onto a population which is already oscillating. We are interested in studying which kind of synchronization can occur between the emitting and receiving populations. It is thought that phase locking properties underlie the basics of neuronal communication [6].

Mathematically, we consider a Wilson-Cowan system [17] properly tuned to show oscillations and we perturb it with a periodic function. In particular, we focus on oscillations arising from a Hopf bifurcation. The periodic forcing of such bifurcation has been studied in classical papers [8], and recently, in the neuroscience context [16]. Here, we consider the stroboscopic map and apply powerful computational methods recently reviewed in [9] to compute the invariant curves and dynamics on them. This allows us to understand how the perturbed invariant curves evolve as the perturbation parameters (frequency and amplitude) are varied, providing a framework that enlarges the comprehension of the dynamics (phase-locking regimes) generated by the periodic forcing. 


\section{Mathematical Model}

A neuronal population can be modelled as a neuronal network consisting of $\mathrm{N}$ neurons connected through synapses. If each neuron is described by a system of $n$ differential equations, a network of $N$ neurons will be described by a system of $N \cdot n$ equations. This approach, although generates very accurate results, considers a high dimensional system, that requires a high computational effort to integrate it and makes the mathematical analysis difficult. As an alternative to this approach, there exist mean field models, which describe spike population rates. These models have a reduced number of equations. One of the most famous rate models are the WilsonCowan equations [17]:

$$
\begin{aligned}
\tau_{e} \frac{d E}{d t} & =-E+\left(1-r_{e} E\right) S_{e}\left(c_{1} E-c_{2} I+P\right), \\
\tau_{i} \frac{d I}{d t} & =-I+\left(1-r_{i} I\right) S_{i}\left(c_{3} E-c_{4} I+Q\right),
\end{aligned}
$$

where the variables $E$ and $I$ correspond to the fraction of neurons of the excitatory and the inhibitory populations which are emitting an action potential at time $t$. The coupling constants $c_{i}$ determine the strength of connexions between neuronal populations. $\mathrm{P}$ and $\mathrm{Q}$ are the (constant) external inputs which are injected to $\mathrm{E}$ and $\mathrm{I}$ populations, respectively (see Fig. 1). Coupling constants $c_{i}$ are positive, whereas external currents $\mathrm{P}$ and $\mathrm{Q}$ can be either positive or negative.

Assuming that a population $k$ is receiving an input $x$, the proportion of cells which will fire as a result of this input is modelled by the response function $S_{k}(x)$, a sigmoidal function defined as:

$$
S_{k}(x)=\frac{1}{1+e^{-a_{k}\left(x-\theta_{k}\right)}}, \quad \text { with } \quad k=e, i,
$$

where the parameters $\theta_{k}$ and $a_{k}$ are the position of the maximum slope and the value of this maximum slope, respectively.

Neurons have a refractory period $r_{k}$ during which they can not respond to external inputs. The factor $\left(1-r_{k} k\right)$ in equations (1), represents the proportion of neurons of the population $k$ which are able to be excited. In [12] it is shown how this term only rescales the parameters on the nonlinearities $S_{k}(x)$ and does not change the

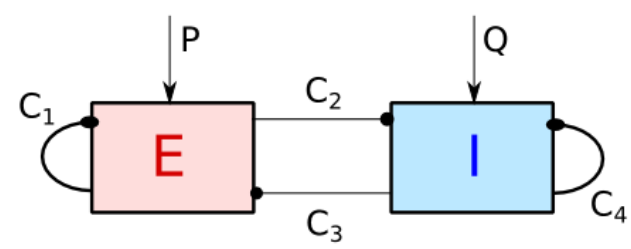

Fig. 1 Schematic of our neuronal network modelled by a mean field model 
qualitative behaviour of the system. For this reason, from now on, we will consider $r_{k}=0$.

\subsection{Dynamical Analysis}

In this subsection we study the most important objects (critical points and limit cycles) of system (1) and their bifurcations. Nullclines of (1), when $r_{e}=r_{i}=0$, are given by

$$
\begin{gathered}
E=f_{e}(I, Q)=\frac{1}{c_{3}}\left[S_{i}^{-1}(I)+c_{4} I-Q\right], \\
I=g_{i}(E, P)=\frac{1}{c_{2}}\left[c_{1} E-S_{e}^{-1}(E)+P\right] .
\end{gathered}
$$

As it can be seen in equations (2), $P$ and $Q$ translate nullclines and thus determine their intersection. Consequently, the name and position of the critical points of the system will depend on the values of $P$ and $Q$. For this reason parameters $P$ and $Q$ will be considered as bifurcation parameters.

Under appropriate conditions, Wilson-Cowan equations are known to show oscillatory behaviour [17]. As we are in a 2D system and phase space is bounded, using Poincaré-Bendixon theorem it is enough to require the system to have an unique unstable critical point to generate a periodic orbit (oscillations).

Such oscillations are going to appear across Hopf, Saddle Node on Invariant Circle (SNIC) and Saddle Node [3,10]. As a first step to find these bifurcations we look for the bifurcations of the critical points of the system. As we consider a 2D system, it is enough to require system (1) to vanish at critical points. Thus, by defining $D X(E, I, P, Q)$ the Jacobian matrix of system (1), we can look for points $(E, I, P, Q)$ satisfying (2) and conditions $\operatorname{Tr} D X(E, I, P, Q)=0$ for the Hopf bifurcation and Det $D X(E, I, P, Q)=0$ for the Saddle Node bifurcation. As we are dealing with non linear equations, the computation of the bifurcation diagram (Fig. 2) requires computational methods for continuation of curves [14].

\section{Non Autonomous Perturbation}

In this section we will study the effects of a T'-periodic non-autonomous perturbation onto the Wilson-Cowan equations (1). Concretely we will study the following model:

$$
\begin{aligned}
\tau_{e} \frac{d E}{d t} & =-E+S_{e}\left(c_{1} E-c_{2} I+P+A p(t)\right), \\
\tau_{i} \frac{d I}{d t} & =-I+S_{i}\left(c_{3} E-c_{4} I+Q\right)
\end{aligned}
$$




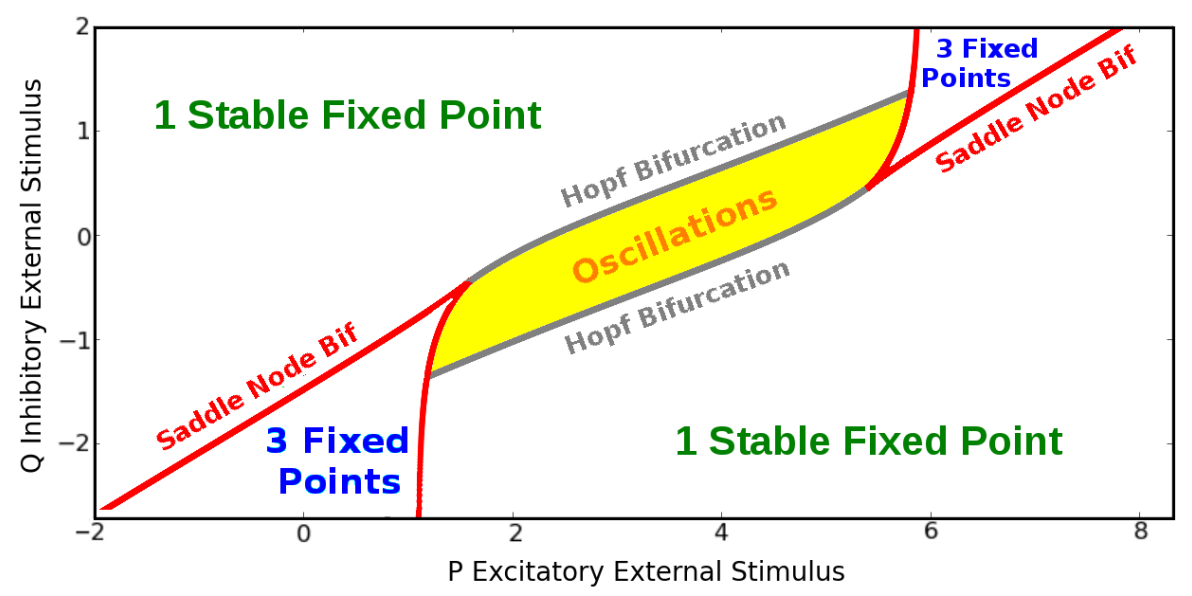

Fig. 2 Bifurcation diagram for the Wilson-Cowan system (1) as a function of external stimulii $P$ and $Q$. Parameters for system (1) are given by the set $\mathscr{P}: c_{1}=13, c_{2}=12, a_{e}=1.3, \theta_{e}=4, r_{e}=$ $0, \tau_{e}=1, c_{3}=6, c_{4}=3, a_{i}=2, \theta_{i}=1.5, r_{i}=0, \tau_{i}=1$

where $A$ is the amplitude of the perturbation and $p(t)$ is the T'-periodic function:

$$
p(t)=1+\cos \left(\frac{2 \pi t}{T^{\prime}}\right),
$$

modelling the activity of an external excitatory population.

\subsection{The Stroboscopic Map}

The stroboscopic map is the most natural approach when considering the study of $\mathrm{T}$ '-periodic perturbations. It is defined by

$$
\begin{aligned}
F_{A}: \mathbb{R}^{2} & \rightarrow \mathbb{R}^{2}, \\
x & \rightarrow F_{A}(x)=\phi_{A}\left(t_{0}+T^{\prime} ; t_{0}, x\right),
\end{aligned}
$$

where $\phi_{A}\left(t ; t_{0}, x\right)$ is the solution of (3) such that $\phi_{A}\left(t_{0} ; t_{0}, x\right)=x$.

As it is well known, the fixed points and invariant curves of the stroboscopic map (4) correspond to periodic and quasiperiodic solutions of system (3) respectively. For instance, if $\gamma(t)=\phi_{A}\left(t ; t_{0}, x\right)$ is a solution of system (3) and $\left[F_{A}(x)\right]^{q}=x$, then by definition $\phi_{A}\left(t_{0}+q T^{\prime} ; t_{0}, x\right)=x$ and therefore $\gamma(t)$ is periodic of period $q T^{\prime}$. Analogously, if $\gamma(t)=\phi_{A}\left(t ; t_{0}, x\right)$ is a periodic orbit of period T of (3) with $\frac{T^{\prime}}{T}=\frac{p}{q}$, $p, q \in \mathbb{N}$, then

$$
\left[F_{A}(x)\right]^{q}=\phi_{A}\left(t_{0}+q T^{\prime} ; t_{0}, x\right)=\phi_{A}\left(t_{0}+p T ; t_{0}, x\right)=x,
$$


that is, fixed points $x$ for the map $\left[F_{A}(x)\right]^{q}$ will correspond to periodic orbits of the system (3). In the neuroscience context, relationship (5) indicates that a $p: q$ phase locking state has been established between the population and the perturbation. In the Wilson-Cowan model this means that the neuronal population variables $\mathrm{E}$ and $\mathrm{I}$ have completed $p$ revolutions in the same time that the perturbation $p(t)$ has completed $q$ revolutions. By contrast, if $\gamma(t)=\phi_{A}\left(t ; t_{0}, x\right)$ is periodic of period T but $\frac{T^{\prime}}{T} \notin \mathbb{Q}$, then $\left[F_{A}(x)\right]^{n} \neq x \forall n \in \mathbb{N}$ and $\left[F_{A}(x)\right]^{n}$ fills densely $\Gamma_{A}=\left\{\overline{\phi_{A}\left(t_{0}+n T^{\prime} ; t_{0}, x\right), n \in \mathbb{N}}\right\}$ which is an invariant curve for $F_{A}(x)$. So, depending on the amplitude A and the period T' of the perturbation, the system in (3) can display either a synchronous $p: q$ regime or an asynchronous regime.

\subsection{Computing the rotation number in the perturbed framework}

In this section we will explain how to compute the rotation number. Consider the set of parameters $\mathscr{P}$ defined in caption of Fig. 2 , and let $(P, Q)=(2.5,0)$. As bifurcation diagram on Fig. 2 shows, for this set of parameters the unperturbed Wilson-Cowan equations (1) display an unstable focus $P_{1}$ and an attracting limit cycle $\gamma$ of period $T \approx 5.26$.

When $A=0$ the phase portrait described by the stroboscopic map (4) is the same as the one generated by the unperturbed system (1). In particular, $F_{0}\left(P_{1}\right)=P_{1}$ and $F_{0}(\gamma) \subseteq \gamma, \forall T^{\prime}$, that is, $P_{1}$ and $\gamma$ are an unstable fixed point and an attracting invariant curve for the map (4), respectively. When applying a T'-periodic continuous perturbation, as both objects $P_{1}$ and $\gamma$ are normally hyperbolic, they will continue existing for weak enough perturbations, and they can be studied as invariant objects for the stroboscopic map $F_{A}$ when $A \neq 0$. In particular, while the unstable focus $P_{1}=P_{1}(A)$ will remain a fixed point for the stroboscopic map $F_{A}$, the attracting limit cycle $\gamma$ will become an attracting invariant curve $\Gamma_{A}$. Over the invariant curve $\Gamma_{A}$ we can define and compute the rotation number.

The rotation number is is defined for any orientation preserving map of the circle

$$
\begin{aligned}
f: \mathbb{T} & \rightarrow \mathbb{T} \\
\theta & \mapsto f(\theta)
\end{aligned}
$$

as

$$
\rho=\lim _{n \rightarrow \infty} \frac{\theta_{n}-\theta_{0}}{n}, \quad \theta_{n}=f^{n}\left(\theta_{0}\right) .
$$

As it is well known, $\rho$ exists and is independent of the point $\theta_{0}$ [1]. Moreover, if $\rho=\frac{p}{q} \in \mathbb{Q}$, the map $f$ has one periodic point $\boldsymbol{\theta}^{*}$ of period $q$. On the other hand, under some regularity assumptions, if $q \in \mathbb{R} \backslash \mathbb{Q}$, the map $f$ is conjugated to a rotation of angle $\rho$ and the orbit of every point $\theta$ fill densely $\mathbb{T}$.

In our case, one can take $f:=f_{A}=\left.F_{A}\right|_{\Gamma_{A}}$ and compute the rotation number as follows: given a point $x \in \Gamma_{A}$, define the angle $\theta$ between the line from $P_{1}(A)$ to $x$ and 
the positive E-axis. Then, given a point $x_{0} \in \Gamma_{A}$ for $x_{n}=f_{A}^{n}\left(x_{0}\right)$, one can compute the rotation number in (6).

When computing $\rho$ numerically, usually the limit to infinity is substituted by a large enough number of iterations but the convergence to $\rho$ is very slow. We used the methods presented in [13], which refine the computation of rotation numbers saving computational effort and accelerating the convergence of the method.

Recall that if $\rho=\frac{p}{q}$ with $p, q \in \mathbb{N}$ there exists a $\theta^{*}$ such that $f_{A}^{q}\left(\theta^{*}\right)=\theta^{*}$, and the corresponding point $x^{*}$ is a $q$-periodic orbit of $F_{A}$, which turns $p$ times around the invariant curve $\Gamma_{A}$. This indicates the appearance of a given $p: q$ phase locking regime in system (3).

In Fig. 3 we show the computation of the rotation number for some amplitude values $A$ and varying T'. We observe the classical Devil's Staircase function [1]. The function shows intervals on the $x$-axis (showing the ratio $\frac{T^{\prime}}{T}$ ) for which the rotation number $\rho\left(T^{\prime}\right)$ is constant (there exists a solution of (3) which is phase-locked to the periodic perturbation). For small positive amplitudes, the largest intervals correspond to the phase locked states 1:1 $(\rho=1)$ and 1:2 $\left(\rho=\frac{1}{2}\right)$ (Fig. 3 top). The phase-locked intervals widen as the amplitude is increased (Fig 3 bottom). The rotation number displays a discontinuity at some values of $\mathrm{T}$ ' jumping suddenly to $\rho=1$ as the amplitude is increased. As the rotation number is defined over the invariant curve $\Gamma_{A}$, this discontinuity may indicate a bifurcation or the breakdown of the invariant curve appearing for non-weak amplitudes that we will investigate in the next sections.
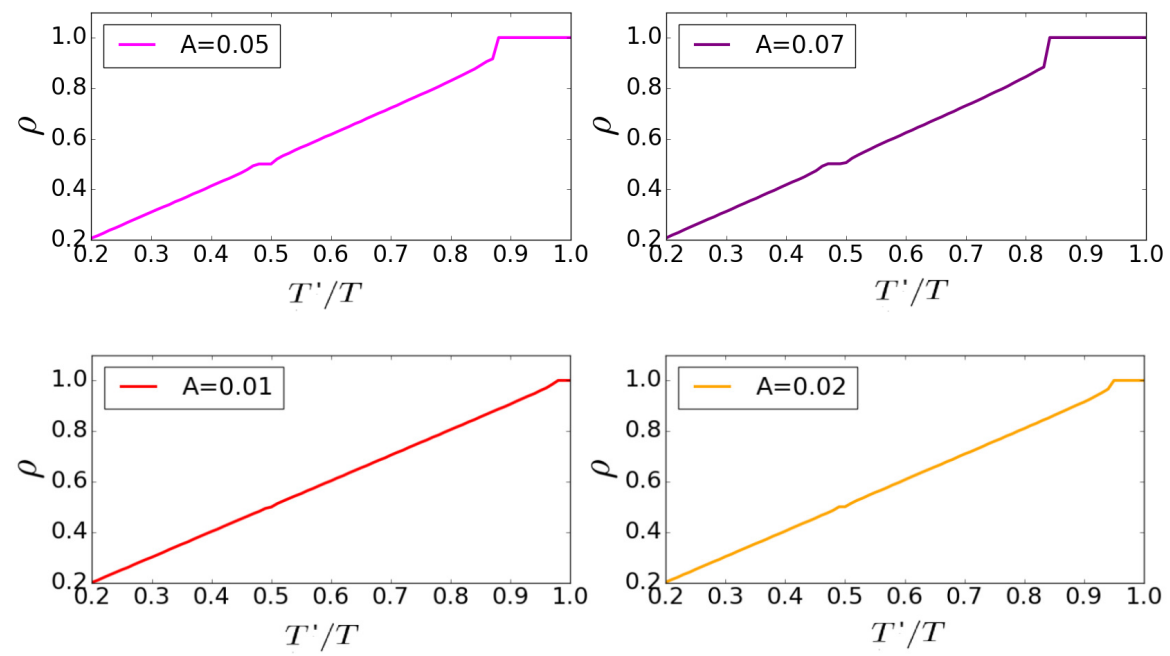

Fig. 3 Rotation number computed for different amplitudes in the perturbed Wilson-Cowan model (3). Parameters used were the set $\mathscr{P}$, and $(P, Q)=(2.5,0)$ 


\subsection{Bifurcation Analysis}

In order to understand the dynamics that occur in system (3) depending on the period T' and the amplitude A of the perturbation, we will begin by computing bifurcations for the fixed points of the stroboscopic map (4).

Given a map $F: \mathbb{R}^{n} \times \mathbb{R} \rightarrow \mathbb{R}^{n}$ depending on one parameter $\alpha \in \mathbb{R}$ :

$$
(x, \alpha) \rightarrow F(x, \alpha), \quad \alpha \in \mathbb{R}
$$

If there exists $\left(x_{0}, \alpha_{0}\right)$ such that

1. $F\left(x_{0}, \alpha_{0}\right)=x_{0}$

2. $D F\left(x_{0}, \alpha_{0}\right)$ has eigenvalues $\lambda$ with $|\lambda| \neq 1$,

then $x_{0}$ is called a hyperbolic fixed point and it is known that for $\alpha \simeq \alpha_{0}$, there exists $x_{\alpha}$ fixed point of $F\left(x_{\alpha}, \alpha\right)$ of the same topological type of $x_{0}$. Otherwise, when 2) fails we call $\alpha_{0}$ a bifurcation value.

Thus, bifurcation values of the map $F$ must satisfy being fixed points of the map (7) and also a bifurcation condition $\Phi_{B I F}$. Mathematically,

$$
G(x, \alpha)=\left\{\begin{array}{l}
F(x, \alpha)-x=0 \\
\Phi_{B I F}(x, \alpha)=0 .
\end{array}\right.
$$

We look for bifurcations of the fixed points of the map defined in (5) for $q=1$. We found two bifurcations of fixed points (see Fig. 4): Saddle-Node ( $S N)$ and NeimarkSacker $(N S)$. At it is well known, a SN bifurcation occurs when one of the real eigenvalues for the fixed point equals one, whereas a NS bifurcation occurs when a fixed point has a pair of complex eigenvalues whose modulus equals one. In a $2 \mathrm{D}$ system the conditions which must be satisfied at these bifurcation values are written as

$$
\begin{aligned}
& \Phi_{S N}=\operatorname{det}(D F-I d)=0 \\
& \Phi_{N S}=\operatorname{Tr}(D F)-1=0
\end{aligned}
$$

where we denote by $D F$ the Jacobian matrix of the map $F$ evaluated at the fixed point, whose computation requires the integration of a second order variational system [14].

Computations depicted in Fig. 4 also enlight the rotation number results shown in Fig. 3. One guesses that discontinuities on the rotation number in Fig. 3 can be caused by the disappearance of the invariant curve (which exists for small amplitudes) across a Neimark-Sacker bifurcation. To give a complete description of the dynamics, besides the computation of the fixed points and its bifurcations we are going to compute the invariant curves to check its persistence and relate its disappearance with the discontinuities observed in the rotation number curves. 


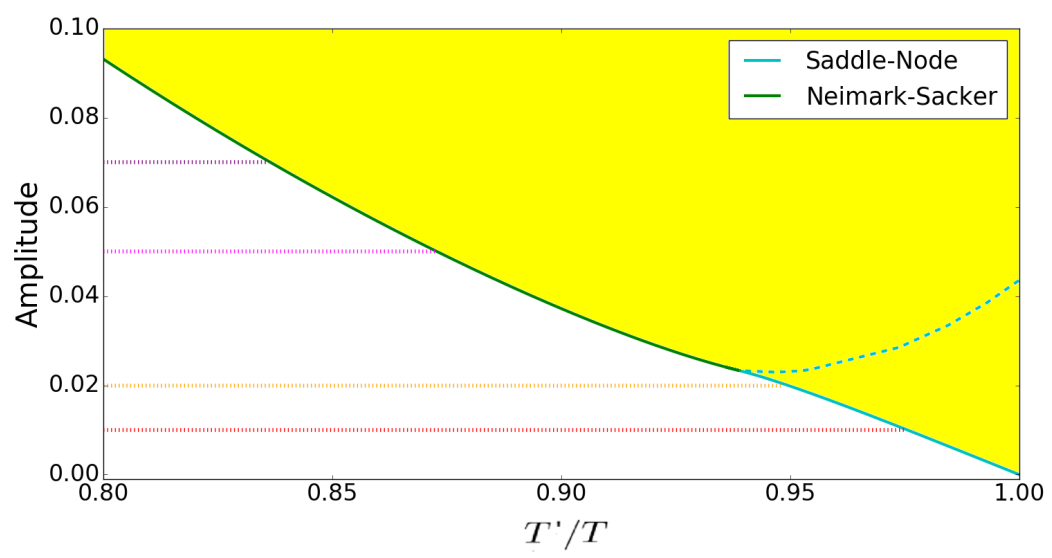

Fig. 4 Bifurcation diagram for the stroboscopic map $F_{A}$ of the perturbed Wilson-Cowan system (3). Parameters used were the set $\mathscr{P}$ and $(P, Q)=(2.5,0)$. Two bifurcations were found: NeimarkSacker (green curve) and Saddle-Node (cyan curve). The solid line corresponds to a SN between a saddle and a stable node whereas the dashed line corresponds to a $\mathrm{SN}$ between a saddle and an unstable node. Constant amplitudes for which rotation numbers in Fig. 3 were computed were drawn respecting the same color code. Inside the yellow area there exists a stable fixed point fot the map $F_{A}$ corresponding to a 1:1 phase locking relationship

\subsection{Computation of Invariant Curves}

As it was observed in section 3.3, the computation of invariant curves is needed to provide a full description of the dynamics generated by the perturbation in (3). The framework developed in [4] allows us to compute a parameterization of the invariant curve $\Gamma_{A}$ issuing from the unperturbed limit cycle $\gamma$. We now briefly review the method and refer the reader to [4] for a detailed description of the method.

Given a map $F: \mathbb{R}^{2} \rightarrow \mathbb{R}^{2}$ having an invariant curve $\Gamma_{A}$, we look for a parameterization $K: \mathbb{T} \rightarrow \mathbb{R}^{2}$ of this invariant curve by solving the following invariance equation

$$
F(K(\theta))=K(f(\theta)),
$$

where $K(\theta)$ and the dynamics inside the curve $f(\theta)$ are both unknown. Differentiating (9) we find the invariance equation for the tangent bundle $D K(\theta)$ :

$$
D F(K(\theta)) D K(\theta)=D K(f(\theta)) D f(\theta),
$$

and imposing the invariance of the normal (stable) bundle of $K(\theta)$, denoted by $N(\theta)$, we have the following invariance equation

$$
D F(K(\theta)) N(\theta)=N(f(\theta)) \Lambda_{N}(\theta),
$$

where $\Lambda_{N}(\theta)$ denotes the linearised dynamics over $N(\theta)$. 
We introduce the matrices $P(\theta)=(D K(\theta) N(\theta))$, and $\Lambda(\theta)=\operatorname{diag}(D f(\theta)$, $\left.\Lambda_{N}(\theta)\right)$ in order to express in a more compact way the invariance equations (10) and (11):

$$
D F(K(\theta)) P(\theta)=P(f(\theta)) \Lambda(\theta) .
$$

Therefore, if we express the linear map $D F(K(\theta))$ in the basis provided by $P(\theta)$, it becomes diagonal. Taking profit of this adapted invariant frame, a Newton method is performed. As it is usual in Newton methods, we assume given the approximation for the unknowns $K(\theta), f(\theta), N(\theta)$ and $\Lambda_{N}(\theta)$ and we compute better approximations:

$$
\begin{aligned}
\bar{K}(\theta) & =K(\theta)+\Delta K(\theta), \\
\bar{f}(\theta) & =f(\theta)+\Delta f(\theta), \\
\bar{N}(\theta) & =N(\theta)+\Delta N(\theta), \\
\bar{\Lambda}_{N}(\theta) & =\Lambda_{N}(\theta)+\Delta \Lambda_{N}(\theta) .
\end{aligned}
$$

To determine the correction terms $\Delta K(\theta), \Delta f(\theta), \Delta N(\theta), \Delta \Lambda_{N}(\theta)$, the Newton method performed is split in two substeps. In the first one, we look for corrections $\Delta K(\theta)$ and $\Delta f(\theta)$. We begin by substituting expressions (13) and (14) into the invariance equation (9), and then expanding in Taylor series around $K$ and $f$ respectively and neglecting quadratically small terms, we obtain

$$
0=E(\theta)+D F(K(\theta)) \Delta K(\theta)-D K(f(\theta)) \Delta f(\theta)-\Delta K(f(\theta))
$$

where $E(\theta)=F(K(\theta))-K(f(\theta))$ is the error for the approximated solution.

Writing Eq. (17) in the adapted frame provided by $P(\theta)$, that is, writing $\Delta K(\theta)=$ $P(\theta) \xi(\theta)$, we obtain the following cohomological equation

$$
\eta(\theta)=\Lambda(\theta) \xi(\theta)-\xi(f(\theta))-\left(\begin{array}{c}
\Delta f(\theta) \\
0
\end{array}\right)
$$

where $\eta(\theta)=-(P(f(\theta)))^{-1} E(\theta)$ is the error of the approximate solution in the adapted frame. $\eta(\theta)$ is a vector which has tangent and normal components, each of them having different equations

$$
\begin{aligned}
& \eta^{T}(\theta)=\Lambda_{T}(\theta) \xi^{T}(\theta)-\xi^{T}(f(\theta))-\Delta f(\theta), \\
& \eta^{N}(\theta)=\Lambda_{N}(\theta) \xi^{N}(\theta)-\xi^{N}(f(\theta))
\end{aligned}
$$

where unknowns $\xi^{T}$ and $\xi^{N}$, can be computed separately by means of a fixed point method.

So far, we have find the corrections $\Delta K(\theta)$ and $\Delta f(\theta)$. Then one can proceed to the second substep of the Newton method. Analogously to the first substep, by substituting the equations (15) and (16) in the invariance equation (11) and applying the same methodology as in the first substep one can find the new corrections for the normal bundle $\Delta N(\theta)$ and its linearised dynamics $\Delta \Lambda_{N}(\theta)$. See [4] for more details. 
We have reviewed the principal steps of the method. Next we are going to provide some details about the computation of the initial seeds for the Newton method in our problem. For a small perturbation, one can use as initial seed the invariant curve for the unperturbed system. Having an unperturbed system which displays a limit cycle $\gamma(t)$ of period T, we can define $\theta=\frac{t}{T}$ as an angular variable which parameterizes the limit cycle $\Gamma_{0}(\theta)=\gamma(\theta T)$. Therefore, as initial seed for the parameterization $K$ and the dynamics on it when A is small we will take $K_{0}(\theta)=\Gamma_{0}(\theta)$ and $f_{0}(\theta)=\theta+\frac{T^{\prime}}{T}$.

In order to find an initial seed for the normal bundle and its dynamics, we compute the monodromy matrix by integrating the variational equations on the limit cycle and $\gamma(t)$ for the unperturbed system over a period $\mathrm{T}$. The diagonalization of the monodromy matrix provides exactly the solutions for the system (11). In particular, as the eigenvector associated to the tangent direction will have trivially an eigenvalue equal to 1 , it is easy to distinguish the normal bundle $N(\theta)$ component and its eigenvalue $\Lambda_{N}(\theta)$ (the characteristic Floquet multiplier), whose value is constant and thus independent of $\theta$.

In order to explain rotation number discontinuities we apply this method to compute invariant curves for system (3) which complete the bifurcation diagram analysis in Fig. 4. If we fix $\frac{T^{\prime}}{T}=0.965$ we expect to cross a SN bifurcation for an amplitude $A_{S N} \simeq 0.014$. This is exactly what Fig. 5 shows: for a value of $A=0.01<A_{S N}$ there exists an invariant curve whose dynamics have no crossings with the fixed points of $f(\theta)=\theta$. By contrast for $A=0.02>A_{S N}$ two crossings appear between $f^{A}(\theta)$ and $f(\theta)=\theta$ indicating the presence of two fixed points over the invariant curve. This can be seen in another way when looking at the rotation number results: for $A=0.01$ rotation number was different from 1 , whereas it was equal to 1 for $A=0.02$ which showed fixed points.

By contrast when fixing $\frac{T^{\prime}}{T}=0.85$ a NS bifurcation is expected to be crossed at $A \simeq 0.062$. This is exactly what Fig. 6 show: as the amplitude is increased, the invariant curve shrinks, but there are no crossings between the dynamics $f^{A}(\theta)$ and the line of fixed points. These results are consistent with rotation number results in Fig. 3. When looking at values of $\rho$ at $\frac{T^{\prime}}{T}=0.85$, when $A=0.05<A_{N S}$ a rotation number different from one appears as it is expected in an invariant curve with no fixed points over it. By contrast, for $A=0.07>A_{N S}$ as there is no invariant curve, rotation number calculations do not work. Nevertheless, a value for $\rho(0.85)=1$ was computed. We think that this result is a consequence of having a fixed point dynamics calculated assuming an orientation preserving map is defined. So, although we tend to a fixed point we assume that each iteration of the map gives a complete revolution before returning to the fixed point.

\section{Dynamics of the Stroboscopic Map}

Section 3 was devoted to the introduction of the stroboscopic map, its invariant objects and its bifurcations, providing techniques to compute all of them. In this section we aim at using all the tools provided in section 3 to give a full descrip- 

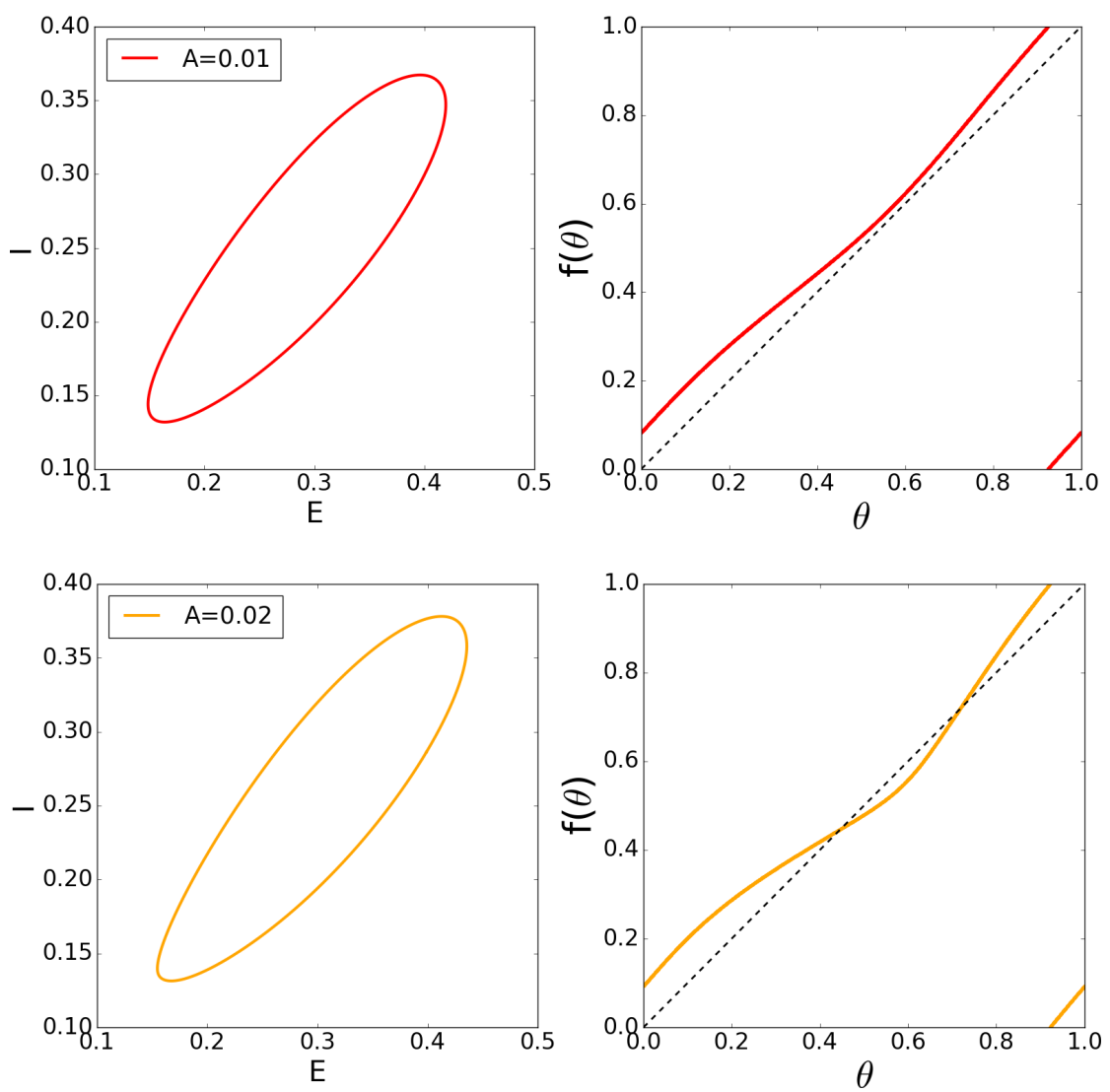

Fig. 5 (Left) Invariant curve for the stroboscopic map with different amplitudes and $\frac{T^{\prime}}{T}=0.965$. (Right) Dynamics $f(\theta)$ over the invariant curve

tion of the stroboscopic map dynamics for the perturbed system (3) close to the 1:1 phase-locking area by describing the evolution of all invariant objects of the system. Finally, will distinguish asynchronous from synchronous areas and study its implications for neuroscience.

\subsection{Phase Space Analysis}

As the bifurcation analysis in Fig. 4 shows, there exist two possible bifurcations depending on the period T' and the amplitude of the perturbation: a Neimark-Sacker (NS) and a Saddle-Node (SN) bifurcation. Moreover, a Bogdanov-Takens bifurcation occurs at $\left(\mathrm{A}, \frac{T^{\prime}}{T}\right) \simeq(0.023,0.9388)$ delimiting the NS and $\mathrm{SN}$ bifurcation 

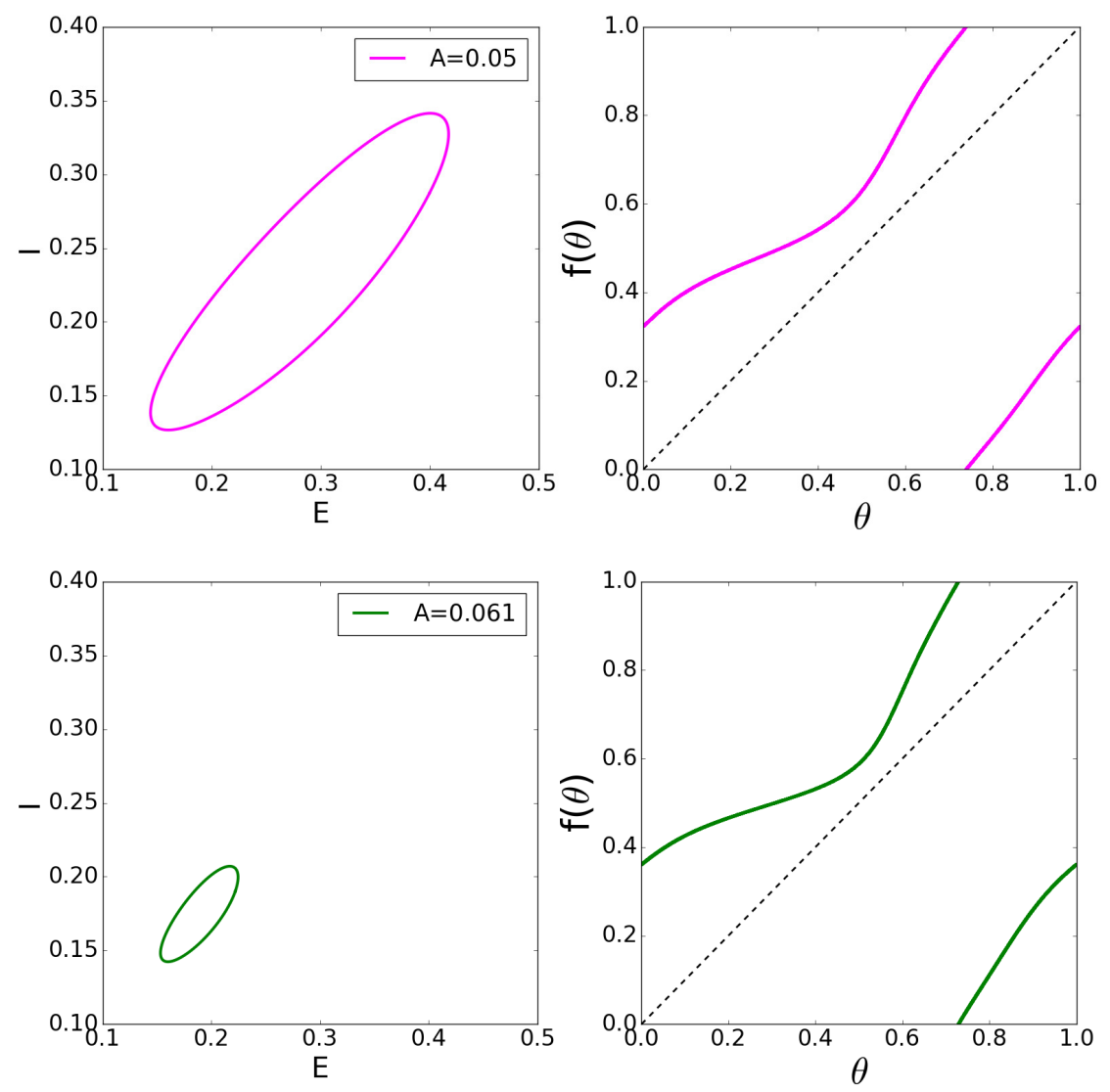

Fig. 6 (Left) Invariant curve for the stroboscopic map with different amplitudes and $\frac{T^{\prime}}{T}=0.85$. (Right) Dynamics $f(\theta)$ over the invariant curve

curves. As different bifurcations will generate different dynamics, we will present phase portraits for the stroboscopic map at the crossing of both bifurcations in order to provide a description of the dynamics of the system (3) close to resonance $1: 1$. More precisely, we will restrict the analysis of dynamics to the range of $\frac{T^{\prime}}{T}$ values that we have shown in Fig. 4, this is from 0.8 to 1 where the rotation number presented discontinuities.

For values of T' such that $0.9388<\frac{T^{\prime}}{T}<1$, the phase portrait for system (3) can be seen in Fig. 7. In region $A_{1}$, the attracting invariant curve $\Gamma_{A}$ generated from unperturbed limit cycle $\gamma$ has no fixed points of $F_{A}$, and an unstable focus $P_{1}$ exists inside $\Gamma_{A}$. Once the saddle-node bifurcation (solid blue line) is crossed (region B), there appear two fixed points on the invariant curve $\Gamma_{A}$ : a stable node $P_{2}$ and a saddle $P_{3}$. The invariant curve consists of the union of the saddle $P_{3}$, its unstable invariant manifolds, and the stable node $P_{2}$. When increasing the amplitude (region $\mathrm{C}$ ), $P_{1}$ 

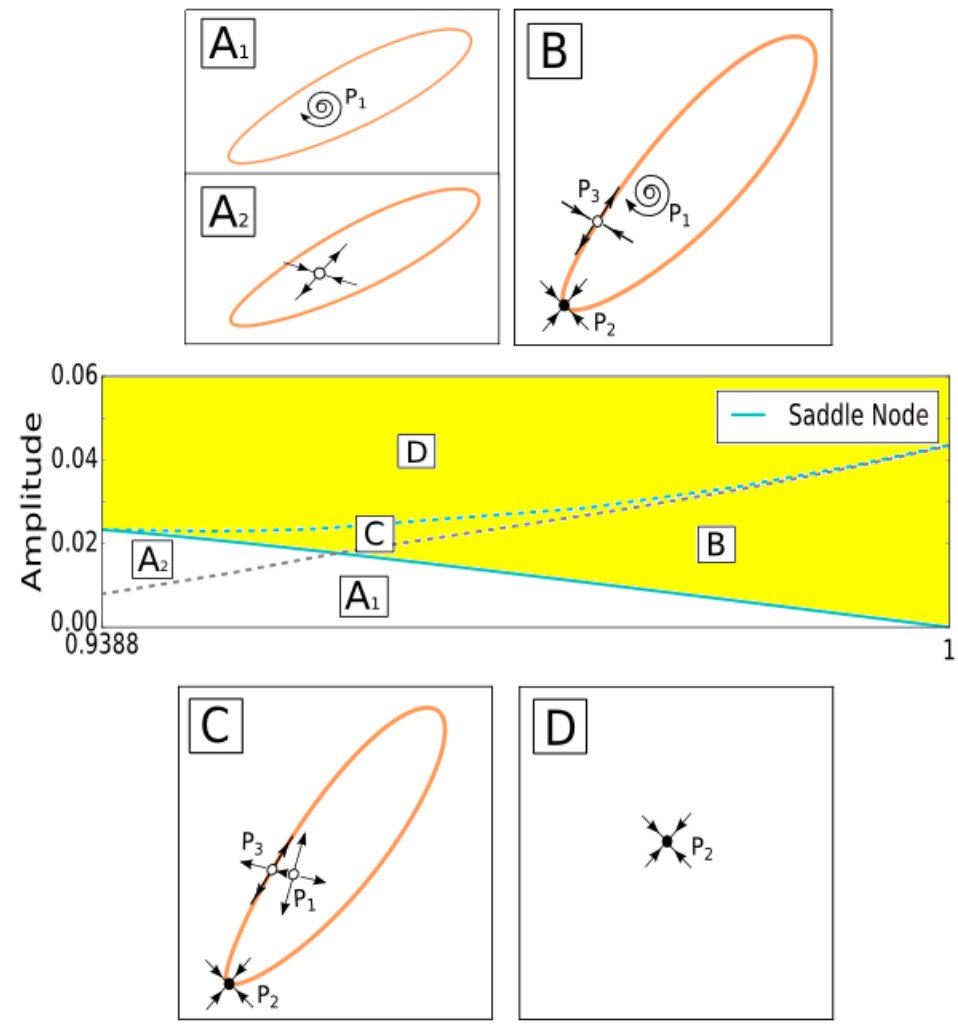

Fig. 7 Dynamics when a Saddle-Node bifurcation is crossed. Fixed points and invariant curves were computed using provided numerical methods

becomes an unstable node (dashed gray line). If the amplitude is increased further, $P_{1}$ will coalesce with $P_{3}$ in a unstable saddle-node bifurcation (dashed blue line) leaving the stable node $P_{2}$ as the unique fixed point (region D). As one may note it is possible to pass from area $A_{1}$ to area $\mathrm{C}$, without passing from $\mathrm{B}$. When entering in the area $A_{2}$ the unstable focus $P_{1}$ can become an unstable node before crossing the SN bifurcation.

For values of T' such that $0.8<\frac{T^{\prime}}{T}<0.9388$, the phase portrait for system (3) can be seen in Fig. 8. The attracting invariant curve $\Gamma_{A}$ has no fixed points of $F_{A}$, and an unstable focus $P_{1}$ exists inside $\Gamma_{A}$ (region A). As the amplitude $A$ is increased, this situation persists until we reach the Neimark-Sacker bifurcation (green curve), where the curve $\Gamma_{A}$ collapses with $P_{1}$ and disappears while $P_{1}$ becomes a stable focus (region B).

Phase space analysis performed gives a fully understanding of the dynamics for the perturbed system (3), demonstrating the presence of a fixed point for the map 


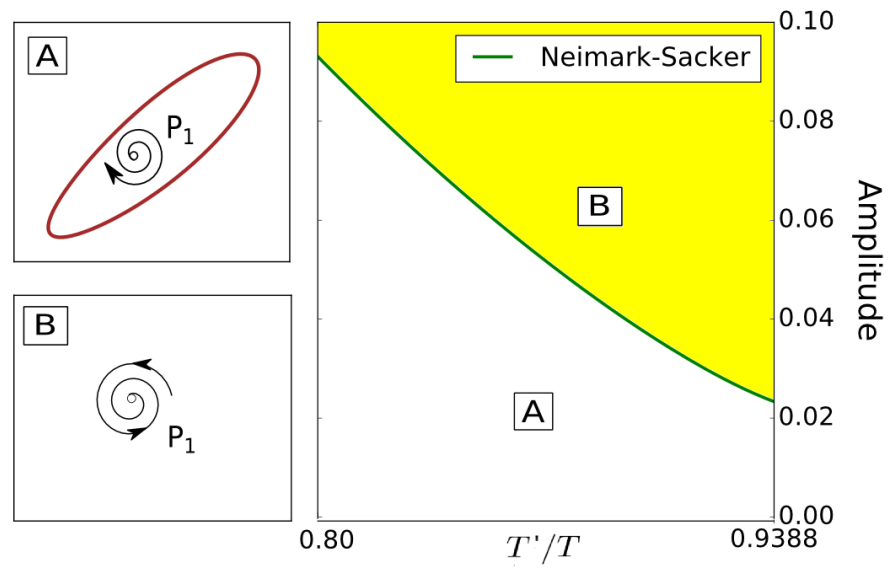

Fig. 8 Dynamics when a Neimark-Sacker bifurcation is crossed. Fixed points and invariant curves were computed using provided numerical methods

$F_{A}$ and thus explaining discontinuities and results for rotation number. For a given amplitude, discontinuities in the rotation number are expected to appear for the exact value of $\frac{T^{\prime}}{T}$ for which a NS bifurcation appears.

\subsection{From synchronous to asynchronous behaviour}

As fixed points for the stroboscopic map correspond to periodic orbits of the system (3), the disappearance of stable fixed points across bifurcations separates the synchronous from the asynchronous regime. Computing the bifurcation curves of system (3) is the most natural way for delimiting and studying a given phase locking relationship. In Fig. 9 we show the stable solutions for a synchronous and an asynchronous state. It can be seen how the phase or time lag between the system and the perturbation is constant in the synchronous regime whereas it is not the case in the asynchronous.

As various theories suggest synchrony between oscillating activity of two neuronal populations may have very important implications in neural communication [11]. In particular, this time lag difference may underlie a possible mechanism for selection of transmitted information [7]. 

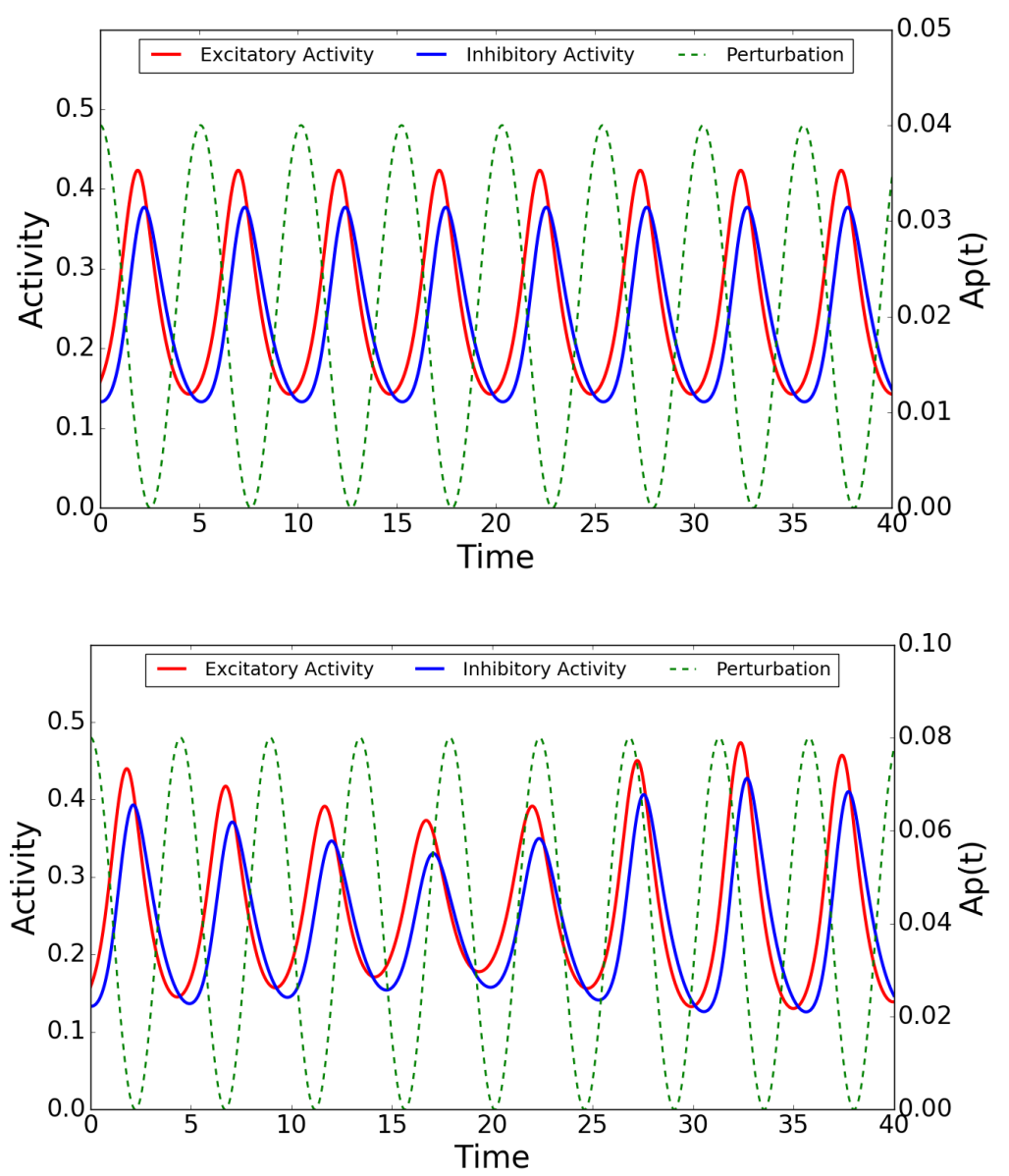

Fig. 9 (Top) Synchronous solution of system (3) for $A=0.02$ and $\frac{T^{\prime}}{T}=0.965$. (Bottom) Asynchronous solution of system (3) for $A=0.04$ and $\frac{T^{\prime}}{T}=0.85$

\section{Summary}

We have considered a periodic perturbation of the Wilson-Cowan equations and we have looked for synchronous and asynchronous regimes. In particular, we have studied phase locking relationships through the rotation number. Computations of this magnitude showed discontinuities which have been understood through the computation of the main invariant objects (fixed points and invariant curves) of the stroboscopic map close to the resonance $1: 1$. We have shown how powerful computational methods for invariant curves provide a further understanding of the dynamics gen- 
erated by a periodic perturbation. Thus, this work aims at providing powerful tools to study interactions of brain rhythms in the brain.

\section{Acknowledgements}

A.P, G.H and T.S acknowledge financial support from the Spanish MINECOFEDER Grants MTM2012-31714, MTM2015-65715-P and the Catalan Grant 2014SGR504.

\section{References}

1. Arrowsmith, D.K., Place, C.M.: An introduction to dynamical systems. Cambridge University Press (1990)

2. Berger, H.: Über das elektrenkephalogramm des menschen. European Archives of Psychiatry and Clinical Neuroscience 87(1), 527-570 (1929)

3. Borisyuk, R.M., Kirillov, A.B.: Bifurcation analysis of a neural network model. Biological Cybernetics 66(4), 319-325 (1992)

4. Canadell, M., Haro, A.: Parameterization method for computing quasi-periodic reducible normally hyperbolic invariant tori. In: Advances in Differential Equations and Applications, pp. 85-94. Springer (2014)

5. Dyan, P., Abbott, L.: Theoretical neuroscience. computational modeling of neural systems (2001)

6. Fries, P.: A mechanism for cognitive dynamics: neuronal communication through neuronal coherence. Trends in cognitive sciences 9(10), 474-480 (2005)

7. Fries, P., Reynolds, J.H., Rorie, A.E., Desimone, R.: Modulation of oscillatory neuronal synchronization by selective visual attention. Science 291(5508), 1560-1563 (2001)

8. Gambaudo, J.M.: Perturbation of a hopf bifurcation by an external time-periodic forcing. Journal of differential equations 57(2), 172-199 (1985)

9. Haro, À., Canadell, M., Figueras, J.L., Luque, A., Mondelo, J.M.: The Parameterization Method for Invariant Manifolds. Springer (2016)

10. Hoppensteadt, F.C., Izhikevich, E.M.: Weakly connected neural networks, vol. 126. Springer Science \& Business Media (2012)

11. Niebur, E., Hsiao, S.S., Johnson, K.O.: Synchrony: a neuronal mechanism for attentional selection? Current opinion in neurobiology 12(2), 190-194 (2002)

12. Pinto, D.J., Brumberg, J.C., Simons, D.J., Ermentrout, G.B., Traub, R.: A quantitative population model of whisker barrels: re-examining the wilson-cowan equations. Journal of computational neuroscience 3(3), 247-264 (1996)

13. Seara, T.M., Villanueva, J.: On the numerical computation of diophantine rotation numbers of analytic circle maps. Physica D: Nonlinear Phenomena 217(2), 107-120 (2006)

14. Simó, C.: On the analytical and numerical approximation of invariant manifolds. In: Les Méthodes Modernes de la Mécanique Céleste. Modern methods in celestial mechanics, vol. 1, pp. 285-329 (1990)

15. Tiesinga, P.H., Sejnowski, T.J.: Mechanisms for phase shifting in cortical networks and their role in communication through coherence. Frontiers in human neuroscience 4, 196 (2010)

16. Veltz, R., Sejnowski, T.J.: Periodic forcing of inhibition-stabilized networks: Nonlinear resonances and phase-amplitude coupling. Neural computation (2015)

17. Wilson, H.R., Cowan, J.D.: Excitatory and inhibitory interactions in localized populations of model neurons. Biophysical journal 12(1), 1-24 (1972) 